\title{
Characteristics of Electrohydrodynamic Roll Structures in Laminar Planar Couette ow
}

\author{
Agisilaos Kourmatzis ${ }^{1}$ and John S. Shrimpton ${ }^{2}$ \\ 1: Department of Engineering, Macquarie University, Sydney, NSW 2109, Australia \\ 2: Energy Technology Research Group, School of Engineering Sciences, University of \\ Southampton, SO171BJ, UK \\ E-mail: agi.kourmatzis@mq.edu.au
}

A bstract.

The behaviour of an incompressible dielectric liquid subjected to a laminar planar Couette ow with unipolar charge injection is investigated numerically in two dimensions. The computations show new morphological characteristics of roll structures that arise in this forced electro-convection problem. The charge and velocity magnitude distributions between the two parallel electrodes are discussed as a function of the top wall velocity and the EHD Rayleigh number, $T$ for the case of strong charge injection. A wide enough parametric space is investigated such that the observed EHD roll structures progress through three regimes. These regimes are de ned by the presence of a single or double-roll free convective structure as observed elsewhere [1], a sheared or stretched roll structure, and nally by a regime where the perpendicular velocity gradient is su cient to prevent the generation of a roll. These three regimes have been delineated as a function of the wall to ionic drift velocity $U_{W}=E$, and the $T$ number. In the stretched regime, an increase in $U_{W}=E$ can reduce charge and momentum uctuations whilst in parallel de-stratify charge in the region between the two electrodes. The st retched roll regime is al so characterised by a substantial in uence of $U_{W}=E$ on the steady development time, however in the traditional non-stretched roll structure regime, no in uence of $U_{W}=E$ on the development time is noted. 
1. Int roduction

NOTICE: this is the authors version of a work that was accepted for publication in the Journal of Physics D: Applied Physics, IOP Publishing. Changes resulting from the publishing process, such as peer review, editing, corrections, structural formatting, and other quality control mechanisms may not be re ected in this document.

Electrohydrodynamics (EHD) is the interdisciplinary area dealing with the interaction betwen hydrodynamic and electrical forces [2]. The eld has numerous industrial applications that range from heat transfer enhancement $[3,4]$, electrostatic spraying applications $[5,6,7,8]$, and electrostatic precipitators [9, 10, 11]. Here, discussion is restricted to dielectric liquids, where charged dipoles and monopoles are separated by neutral entities [12], and electric charge resides in the liquid volume.

Previous studies in 'fre-convection' electrohydrodynamics, where no imposed bulk mean ow is present have largely concentrated on linear instability analysis in order to de ne instability thresholds $[2,13,14]$ above which roll structures manifest in a way partly analogous to thermal natural convection [3, 4]. The precise mechanisms leading to elect ro-convective roll formation have ben described at length in [14] and [13]. Investigations by Atten [14], Schneider and Wat son [15], Hop nger and Gosse [16] and Cast ellanos and co-workers [2, 13], whilst focusing on the onset of instability have also shed light on how electrical forces couple to the hydrodynamic forces. Recent detailed computational investigations have been conducted $[17,18,19]$ that have also focused on analysing the formation of the roll structures which has included spatial distributions of charge and velocity magnitude as well as temporal variations in these quantities [1, 20]. Strong and weak injection regimes have been identi ed and investigated, where this terminology refers to the 'strength' of charge injection, related to the value of charge 
density at the injecting electrode $[1,20]$, which in turn is related to a ' $C$ ' parameter, to be described fully in the section to follow. In the context of forced convection, where a mean ow is present, it is anticipated that the roll structures that arise will take on a di erent form and this is of particular importance in charge injection atomizers [21, 22] where space charge injection occurs perpendicular to a mean ow.

Available literature on forced electro-convection has largely focused on instability analysis in EHD Taylor-Couette ow concentrating both on interfacial analysis betwen liquid layers whilst also single liquid studies [23, 24, 25, 26, 27], and Poiseulle ows [28, 29]. Castellanos and Agrait [30] derived instability thresholds for a 'moderate' injection strength case and de ned the critical instability parameter, or electrical Rayleigh number $T$, as a function of Reynolds number using instability analysis. It was determined that for low Reynolds numbers, EHD instability is dominant and inertial forces generally had a stabilizing e ect [30]. This work was later extended by Lara et al. [27] to include a range of injection strengths and it was found that the onset of instability had a dependence on the 'M' number which is related to the viscosity and electrical permittivity of the liquid.

In the case of Taylor-Couet te ow, instability onset was also found to depend on the location of charge injection, e.g. the destabilizing e ect of electric charge via a Coulomb force is more signi cant when charge is injected int o the liquid from the outer, moving electrode [27]. Similar instability analysis was carried out for the case of Poisseuile ow [28, 29] again for a range of $M$ numbers and injection strengths. The instability criteria for cases of forced ow are signi cantly more complicated $[27,28,29,30]$ than fre convection cases, where the latter are only de ned by the instability parameter $\mathrm{T}$ and the injection strength parameter $\mathrm{C}$. In the former, hydrodynamic dimensionless numbers must also be considered. 
Whilst substantial work has ben done in instability analysis, there is a lack of understanding of thein uence of a forced ow on the roll morphology in EHD convection. Subsequently, it is largely unknown what the in uence of mean convective velocities on the re-distribution of space charge and momentum in the domain is. These are critical areas in the cont ext of designing forced charge injection systems which have applications in electrostatic atomization and heat transfer enhancement $[4,3,31,32,33,34]$. In this contribution, we examine a fundamental parallel-plate EHD problem where the top grounded electrode is moving at a wall velocity relative to the adjacent row of control volumes. The aim of the work is to examine the electro-convective roll structure morphology as a function of both the electrical Rayleigh number, $\mathrm{T}$, and the upper wall velocity $U_{W}$, the latter of which has been scaled on the ionic drift velocity, $E$. The routine utilized has been fully proven for use in strong injection electroconvection [35]. The work to be present ed here can be considered as an extension of the work of Vazquez et.al. [1, 20] where here we expect to reproduce the double-roll structure, and also examine how the electroconvective structures change as a function of $T$ and $U_{W}=E$. In the context of charge injection atomizers, where the internal geometry has ben found to dictate the speci $c$ charge of the spray [21], obtaining an improved understanding of charge distribution between two electrodes, for the case of a forced ow, is of great interest.

The paper is organized as follows: we rstly provide the governing equations along with results which validate the operation of the software for forced electroconvection. Whilst aspects of such validation have already been provided elsewhere [35] some further results are provided here for the bene $t$ of the reader. Following presentation of validation, qualitative changes in the double roll structures as a function of $T$ and $U_{W}$ are discussed and roll structures are qualitatively mapped according to their 
general features. This is complemented by a quantitative investigation of the temporal and spatial distributions of space charge and velocity magnitude. The translational progression of roll structures across the domain is then investigated as a function of $U_{W}=E$ and $T$ and this is followed by an analysis of development times and of charge and momentum uctuations.

\section{Problem formulation}

\subsection{Physics and Governing Equations}

Charge transport is due to di usion, convection, and ionic drift where the latter is the velocity of the liquid due to an electric potential gradient. Here, $\mathrm{V}$ is the voltage (volts), $U$ the uid velocity $(\mathrm{m} / \mathrm{s}), Q$ is the space charge $(\mathrm{C} / \mathrm{kg}), \mathrm{t}$ is the time $(\mathrm{s})$, and is the permittivity $(\mathrm{F} / \mathrm{m})$. Using this scaling, the non-dimensional governing equations for EHD for a unipolar charge species in a pure incompressible ow are given here with reference values as described elsewhere [35] ignoring electrical di usion since the electrical Schmidt number $\mathrm{SC}_{E}>>1$ [2]. These equations have been fully described elsewhere [2, 34], however are reproduced here for the reader's bene $t$ :

$$
\begin{aligned}
& \frac{@}{\bigotimes_{i}}\left(U_{i}\right)=0 \\
& \frac{@}{@}\left(U_{i}\right)+\frac{@}{@ j}\left(U_{i} U_{j}\right)=\frac{1}{R e} \frac{@}{Q_{j}} \frac{@ V_{i}}{\bigotimes_{j}} \quad \frac{@}{@ i}+\frac{G r_{E}}{R e^{2}} Q E_{i} \\
& \frac{@}{@}(Q)+\frac{@}{\bigotimes_{i}}\left(U_{i} Q\right)=\frac{{ }_{0} E_{0}}{U_{0}} E_{i} \frac{@}{\bigotimes_{i}}+Q^{2} \\
& \frac{@}{\bigotimes_{i}} \frac{@ !}{\bigotimes_{i}}=Q C
\end{aligned}
$$


where

$$
\mathrm{Gr}_{\mathrm{E}}=\frac{{ }_{0}^{2} \mathrm{Q}_{0} \mathrm{~V}_{0} \mathrm{~d}_{0}^{2}}{{ }_{0}^{2}} ; \quad \operatorname{Re}=\frac{{ }_{0} \mathrm{U}_{0} \mathrm{~d}_{0}}{0}
$$

Equations 1 to 4 are the conservation of mass, momentum, space charge, and Poisson equation for the voltage respectively. The reader should note that in equation 2 the EHD source term on the R.H.S is only due to an applied Lorentz force, i.e. electrost rictive and dielectrophoretic forces are not considered as they are negligible for the simulation parameters used [1, 20]. The non-dimensional paramet ers appearing have been fully explained elsewhere [2].

Equations 1 to 4 are non-dimensionalized with separate scales for the uid velocity $U_{0}$ and electrical drift velocity $E_{0}$. In the free ow case $U_{0}$ may be scaled to $E_{0}$ and therefore the EHD momentum conservation equation has a di erent form:

$$
\frac{@}{@}\left(U_{i}\right)+\frac{@}{\bigotimes_{j}}\left(U_{i} U_{j}\right)=\frac{@}{@ i}+\frac{1}{\operatorname{Re}_{E}} \frac{@ U_{i}}{@_{j}}+C M^{2} Q E_{i}
$$

The $C$ parameter characterizes the injection strength referred to in the introduction, which governs how 'strong' or 'weak' an injection is and is de ned by equation 7. A system is said to be under a strong injection regime if $C>>1$ and under a weak injection regime if $\mathrm{C}<<1$. This term is the ratio of the ionic drift timescale ${ }_{d}$ to the Coulombic charge relaxation timescale sc and therefore the smaller sc is, the more intense the injection.

$$
\mathrm{C}=\frac{{ }_{0} \mathrm{Q}_{0} \mathrm{~d}_{0}^{2}}{\mathrm{~V}_{0}}=\frac{\mathrm{d}}{\mathrm{sc}}
$$

where

$$
{ }_{\mathrm{d}}=\frac{\mathrm{d}_{0}^{2}}{{ }_{0} \mathrm{~V}_{0}} \quad \mathrm{sc}=\frac{0}{{ }_{0} \mathrm{Q}_{0}}
$$

and

$$
M=\frac{(=0)^{1=2}}{\operatorname{Re}_{E}} \quad{ }^{1=2} ; \operatorname{Re}_{E}=\underline{V}
$$


The ' $T$ ' parameter is the product of the electrical Grashof number $\mathrm{Gr}_{\mathrm{E}}$ and the electrical PrandtI number $\operatorname{Pr}_{E}$ [22] and may be thought of as an electrical Rayleigh number [13, 2], making it an indicat or of electroconvective inst ability [13, 2].

$$
\mathrm{T}=\frac{\mathrm{V}_{0}}{=\mathrm{Gr}_{\mathrm{E}}} \mathrm{Pr}_{\mathrm{E}}
$$

\subsection{Geometry and numerical procedure}

A two-dimensional Cartesian coordinate system is employed where two lines of length $L$ normal to the y-axis are separat ed by a distance d. The width of a single control volume is referred to as $w_{x}$ and $w_{y}$ in the $x$ and $y$ directions respectively. Boundary conditions were chosen so as to achieve a strong injection simulation with $\mathrm{C}=10$ and $\mathrm{Re}_{\mathrm{E}}=1$, where physical properties, voltage and charge levels were altered in order to achieve a range of $\mathrm{T}$ numbers from 500 to 5000 .

An x line of control volumes $\left(w_{y}>y>0\right)$, is at a xed value of charge, $Q_{B}(C / k g)$ and at a $x$ ed value of voltage, $V_{B}$. The $x$ line at $y=0$ is adiabatic $(d V / d y=0$ and $d Q / d y=0)$. For the Couette ow simulations the top $x$ line $(y=d)$ is a moving wall with boundary conditions $\mathrm{V}_{\mathrm{T}}=0$ and $\mathrm{dQ} \mathrm{Q}_{\mathrm{T}}=\mathrm{dy}=0$ and $\mathrm{U}=\mathrm{U}_{\mathrm{W}}=\mathrm{n} \mathrm{E}$ or $\operatorname{Re}=\mathrm{Re}_{\mathrm{E}}=\mathrm{n}$ where $\mathrm{n}$ can take values of $: 05<\mathrm{n}<60$. All simulations were hydrodynamically laminar with Re ranging from 0.01 to 115 , where $R e={ }_{0} U_{W} d_{0}={ }_{0}$. Both y lines enclosing the simulation domain are periodic boundary pairs.

Additionally, in the EHD Couette ow problem, with an increase in $R e=R e_{E}$, a larger body force term is required to sustain a roll. The body force for forced convection scales with $\mathrm{Gr}_{\mathrm{E}}=\mathrm{Re}^{2}$ and table 1 shows the range of magnitudes invest igated here for a selection of $\mathrm{T}$ numbers.

The conditions here were predominantly chosen so as to allow comparison to existent simulations on roll formation in free electro-convection $[1,20]$. However, recent 


\begin{tabular}{ccccc}
\hline $\mathrm{T}$ & $\mathrm{Re}_{\min }$ & $\mathrm{Re}_{\max }$ & $\left(\mathrm{Gr}_{\mathrm{E}}=\mathrm{Re}^{2}\right)_{\max }$ & $\left(\mathrm{Gr}_{\mathrm{E}}=\mathrm{Re}^{2}\right)_{\min }$ \\
\hline 500 & .01 & 21 & $4: 81 \times 10^{7}$ & 12 \\
750 & .70 & 28 & $1: 50 \times 10^{4}$ & 10 \\
1500 & 5.17 & 47 & $5: 81 \times 10^{2}$ & 7 \\
3000 & 5.10 & 79 & $1: 18 \times 10^{3}$ & 5 \\
5000 & 15.61 & 115 & $2: 13 \times 10^{2}$ & 4 \\
\hline
\end{tabular}

Table 1. Typical dimensionless numbers showing full range of $\operatorname{Re}$ and $\mathrm{Gr}_{\mathrm{E}}=\mathrm{Re}^{2}$ investigated for each $\mathrm{T}$ level $\left(\min\right.$ to $\max$ ). For each case $\mathrm{Re}_{E}=1$ and $\mathrm{C}=10$

work [21] has shown that strong injection $\operatorname{EHD}(\mathrm{C}>>1)$ is a normal mode of operation for charge injection atomizers, where forced electro-convection is the norm, and for many charge injection at omizer cases the hydrodynamic Reynolds numbers is in the range of $\operatorname{Re}<200$, as is the case here. While these simulations aim towards a fundamental understanding of roll formation in forced EHD, they are also of practical relevance in charge injection systems.

2.2.1. Numerical method User de ned functions were incorporated into commercial software (Ansys Fluent v15) in order to incorporate the space charge transport and Poisson equation for the voltage, while script was also written to include a Lorentz source term on the momentum conservation equation in two dimensions. The simulation used a 2nd order implicit nite volume scheme in both time and space. More speci cally, the 2nd order QUICK scheme [36] was used to spatially discretise the moment um and space charge transport equations, while time was discretised using a 2nd order fully implicit method. Pressure was also discretised using a second order method and the SIMPLE method [36] was used to couple the pressure and velocity elds. Default second order implicit schemes were used for both int egration and di usion. This nite volume based numerical procedure has been fully validated for use in strong injection EHD [35], and some further validation results for $2 \mathrm{D}$ simulations will be shown here. Computations of plasmas which also involve high non-linearity, have also been successfully carried out 
using a nite volume method [37].

2.2.2. Spatial and Temporal discretisation The simulation was able to accurately capture the analytical solution of charge injection as well as solution independence from the grid and time-step, and the ability of the results to replicate the analytical solution under speci c circumstances will be shown throughout the paper. The timestep for all simulations was signi cantly smaller than the smallest electrical timescale as in [35] and the Courant number was less than than unity for all simulations. A non-uniform mesh was used near to the injection boundary which accurately recovered the analytical solution of charge, and the overall mesh and time-steps chosen for the simulations follow previous work by these authors [35] which use a Kolmogorov lengthscale as a reference. While the conditions presented here are not turbulent, use of the Kolmogorov scale for mesh sizing is conservative. The mesh size here is of the order of 0.1 where $\quad \frac{2700}{M}{ }^{\frac{3}{8}} \mathrm{M}{\frac{T}{M^{2}}}^{\frac{3}{4}}$ I and I $\quad(M=300)^{1=2} \mathrm{~d}[13]$.

\section{Solution Validation: Free Convection Roll Structures}

The linear threshold for strong injection above which roll structures arise has been determined through these simulations to be equal to $T_{C} \quad 170$, whereas [38] quote $\mathrm{T}_{\mathrm{C}} \quad 171$ and [20] quote $\mathrm{T}_{\mathrm{C}}$ 163. Below this threshold, the charge distribution is de ned through an analytical expression. The $T=200$ case shown in gures 1(a) and 1(b) yields a clear double roll structure, which is in excellent qualitative agrement with $[1,20]$. Increasing to $T=500$ results in a strong but fairly spatially and temporally unstable double roll structure as observed by [1], where higher $\mathrm{T}$ numbers will be examined in the results sections. Whilst Fig. 1(b) may be used to qualitatively compare to results of [1], it is also clear that from a quantitative point of view that the bulk of the roll is e ectively void of charge, where in this instant in time, the charge in the roll 
is a maximum of $3 \%$ of the electrode charge level. Observation of gure 2 shows the distribution of charge for the $\mathrm{T}=200$ strong injection case, plotted alongside the results of [20]. The non-linear region is clearly captured as is the central region, when taking a xed vertical line through one of the two double rolls within the larger single roll structure.

Analysis of the non-dimensional velocity magnitude was also conducted for the $T=200$ case with the same $M$ and $C$ numbers as in [20]. Here, the steady state nondimensional velocity magnitude $U_{\text {mag }}=E$ was found to be equal to approximately $\quad 3: 7$, which is a deviation of $8 \%$ when compared to the PIC method of [20]. Furthermore, in the analysis of [39] it is deduced that in convection dominated regions, such as in the domain centre, the uid velocity must be greater than the ionic drift velocity, and this is observed here.

Along with thelinear inst ability threshold which was accurat ely reproduced, we also note the non-linear threshold de ned by a hysteresis loop, which has been discussed in some detail by [38]. The non-linear criterion suggests that there are small dist urbances existing at instability levels smaller than $T_{C}$. We reproduce the non-linear criterion by taking the statistically stationary $T=200, C=10, M=10$ simulation and re-de ning the boundary conditions in order to achieve a $T=190, C=10, M=10$. The subsequent simulation wasthen run until statistically stationary and this was repeat ed by decreasing the $T$ value by 10 every time until a $T=100$ was reached, whilst keping $C$ and $M$ constant. An evident hysteresis e ect was observed, given that for $\mathrm{T}$ values below $\mathrm{T}_{\mathrm{C}}=170$ the double roll structure was still present, showing that the EHD instability threshold when starting the simulation from a fully developed state, is not the same as when starting from a quiescent state. At $T=120$, the double roll structure lost its coherency becoming a single roll, and at $\mathrm{T}=110$, the roll reduced in size signi cantly. 
At $T=T_{f} \quad 105$ there was no roll structure, and the charge distribution was de ned through an analytical solution of charge injection. [38] found the non-linear threshold associated with the hysteresis e ect to be equal to $T_{f}$ 126. Such a variation in the estimation of instability thresholds is sensitive to di erent numerical methods $[20,38]$, and the one we propose here and used in [35] is consistent.

\section{Roll M orphology of EHD Couette Flow}

\section{1. $E$ ect of $T$ on double roll structure}

Figures $3(a)$ to $3(r)$ show normalized charge distribution and velocity magnitude for the $2 \mathrm{D}$ domain examined here. The double roll structure noted previously by [1] is clearly observable through gure 3(a) while it may also be discerned through gure 3(f ). At higher $T$ numbers, the double roll structure is not as stable. In particular, there are inst ances where the number of rolls changes and this is in direct agreement with previous work for strong injection [1]. Particularly for the $T=1500$ and $T=3000$ cases, it was observed that rolls continuously moved in space. While Vazquez et al. [1] showed that the double roll structure appears for $T$ numbers greater than $T=200$, here we observe that above a $T=750$, the stable double roll struct ure is destroyed due to the constant displacement of roll structures that occurs.

\section{2. $E$ ect of $U_{W}$ on double roll structure}

For any given instability parameter $T$, an increase in $U_{W}$ acts to destroy the large scale electro-convective structure, showing how inertial forces arising from the imposed wall velocity act to stabilize the problem, and this has also ben noted for Taylor-Couette ows [27].

We note three types of electro-convective roll structures in the Couette ow 
problem. The rst regime is that of a standard EHD roll structure, which for $T$ values less than approximat ely $\mathrm{T}=750$ is a stable doubleroll structure, and for $\mathrm{T}$ values larger than this, due to continuous roll displacement, is generally observed to be a single roll structure. As the wall velocity $U_{W}$ increases the internal double roll structure is no longer sustained even for $T<750$ and the rolls are generally stretched towards the direction of the shear ow, such as the $T=1500$ cases presented in gures $3(i)$ and $3(j)$. A feat ure of the roll stretching includes space charge being advected to an adjacent roll. This is particularly clear through gure $3(\mathrm{~g})$ where it can be seen that charge is stripped away from a role such that it interacts with the adjacent role, decreasing the degre of charge strati cation in the center of the domain.

As $U_{W}$ is increased further, a fully stable solution is reached with no roll structures present. In this instance, the charge and velocity magnitude distributions can be determined through analytical expressions for one dimensional charge injection and for standard Couette ow respectively. The absence of rolls exists when the velocity $U_{W}$ is high enough to destroy the instabilities, and this may be seen through gures 3(q) and $3(r)$. These morphological features of the rolls are anticipat ed to have important implications in problems such as heat transfer augmentation [4, 3, 31, 32, 33] and int ernal charge injection atomizer ow [21]. In charge injection atomizers in particular, it is possible that features of inter-electrode mixing due to an imposed forced ow would a ect the charge distribution in the liquid jet [40] which would subsequently impact the droplet size distributions.

\subsection{Mapping of Morphology}

Based on the comput ations carried out, a map can be generat ed showing what conditions must exist in EHD Couette ow for a particular roll structure, or absence thereof, to be observed. Figure 4 plots such an instability map indicating the thre types of 
electroconvective roll structures, where in region 'A' either a st able double roll structure is generally observed $\left(A_{1}\right)$, or a general single roll structure with substantial roll shifting $\left(A_{2}\right)$. In region ' $B$ ' roll stretching is observed, and in region ' $C$ ' no rolls are observed. The relationships are close to linear over the range of parameters investigated, making it straight forward to extrapolate the ow structure charact eristic to cases with higher $\mathrm{T}$ and $\mathrm{U}_{\mathrm{W}}=\mathrm{E}$.

These regimes were additionally examined with respect to the value of $\mathrm{Gr}_{E}=\operatorname{Re}^{2}$ given its appearance in the body force term on the momentum transport equation. The value of $\mathrm{Gr}_{E}=\mathrm{Re}^{2}$ is provided for four extreme points on Fig. 4. For instance, the threshold between regime $B$ and $C$ at $T=200$ occurs at $\mathrm{Gr}_{E}=\operatorname{Re}^{2} \quad 21$ where for values below this no rolls are present and for values above this roll stretching can occur, where the threshold betwen stretching and a doubleroll struct ure occurs at $\mathrm{Gr}_{\mathrm{E}}=\mathrm{Re}^{2}=8400$. This is the minimum dimensionless body force magnitude which is required to sustain a particular electroconvective roll structure.

Having made general observations on the morphology of these roll structures as a function of a variety of parameters, quantitative analysis can be carried out in order to characterise them more rigorously in terms of charge and velocity magnitude distributions and this is discussed next.

5. Spatial charge and velocity distributions

Figure 5 shows the time-averaged space charge distribution for the $\mathrm{T}=500 ; \mathrm{C}=$ $10 ; e_{E}=1$ cases at various values of $U_{W}=E$ or $R e=R e_{E}$ alongside the analytical solution for charge injection with no bulk convection. For values of $U_{W}=E>17$ it is evident that the space charge distribution is de ned by the analytical solution, which neglects the presence of bulk charge convection. This indicates that above these values 
of $U_{W}$, unipolar charge injection does not create an electro-convective structure.

Observing gure $5(b)$ it can be seen that as $U_{W}=E$ increases, the charge is rstly a ected in the region where $y=d>0: 125$, where $y / d=0$ is the emitting electrode and $y=d=1$ is the location of the collecting electrode. This occurs because as the top plate moves with a translational velocity, it does not initially in uence the region of space charge that is adjacent to the emitting electrode. A spatial location of approximately $y / d=0.12$ indicates the edge of the space charge layer adjacent to the emit ting electrode. This space charge layer is clearly visible through any of the contour plots of the previous section. With regards to the in uence of $U_{W}=E$ on the space charge near to the collecting electrode, it is interesting to note that close to the collecting elect rode $(y / d=1)$ where the horizontal velocity component is greatest, the di erence in the mean charge pro le amongst di erent values of $U_{W}$ is not substantially di erent to the variation amongst di erent pro les at $y / d \quad 0: 125 . A U_{W}$ of at least $12 E$ is required in this instance in order to begin a ecting the charge distribution adjacent to the emitting electrode, where the upper arrow of gure 5(b) shows the higher $U_{W}$ pro les beginning to approach the analytical solution for charge.

In gure 6 the analytical solution of the non-dimensional velocity magnitude for plane Couette ow in the absence of charge is provided alongside various other cases. The $y$-axis is plotted on a logarithmic scale for clarity, given the wide range of $U_{W}$ values. As with the charge distributions, the pro les for $U_{W}=E>17$ can be adequat ely represented by an analytical Couette ow solution. Lower $U_{W}$ cases deviate and show much higher velocity magnitudes in the centreline, which is largely attributed to the substantial ionic drift velocity component generated by the rolls. For lower $U_{W}$ values, substantial roll deceleration can be observed near the top wall boundary.

Unlike the mean charge distribution pro les, where no case of $U_{W}=E<17$ showed 
good agreement with the analytical solution near the top boundary, in the case of the non-dimensional velocity magnitude, from $y / d=0.85-1$ it is apparent that an analytical solution is adequate. This indicates that the momentum of the rolls near to the top boundary can be adequately predicted by using the wall velocity, even in the presence of charge injection. This implies that even though the charge distribution is substantially in uenced by the roll charge distribution at $y / d=0.85-1$ as can be seen through gure $5(a)$, the velocity magnitude at that location is linked more to $U_{W}$.

The results shown in this section have ben useful in determining the in uence of $U_{W}$ on the distribution of mean charge and velocity magnitude in the domain. However, a further key aspect of forced-electroconvection is its temporal instability. In particular, the in uence of $U_{W}$ for particular levels of $T$ on the time variation of charge and velocity, and this is examined next.

\section{Temporal charge and velocity variations}

Figures $7(a)$ and 7 (b) show the non-dimensional steady-state development times from the start of the simulations for the space charge and velocity magnitude at $y=0.5 d$ respectively. Both the velocity and charge have been spatially averaged along a plane parallel to the electrodes at that vertical location. In general, both results indicate that under conditions where rolls exist, the wall velocity tends to increase the steady state development time. This trend stops abruptly when $U_{W}=E$ has increased su ciently such that rolls are no longer present, and in that situation the development time drops substantially as the solution becomes governed solely by charge injection and not convection. Increasing the value of $U_{W}$ also tends to smoothen the mean velocity and space charge with time substantially which further shows the stabilizing e ect that a forced ow has on the uctuations of charge and moment um. 
The development times are analysed further through gure 8 which shows the velocity magnitude development time plotted vs normalized wall velocity for a variety of cases. For a given $T$ level, for instance $T=500$, the development time is constant until the wall velocity is su cient enough to a ect the roll morphology. At that point, which for $T=500$ is at $U_{W}=E=2$, the added shear ow has the ect of increasing the development time. This occurs because the forced ow component acts to constantly spread charge, temporarily preventing roll formation, before stability is nally attained. Once the $U_{W}$ threshold is reached which no longer allows for roll formation, then the development time sharply drops to a value that tends to be below the ionic drift timescale for the range of cases studied here. The vertical lines on gure 8 indicate this nal transition for the cases investigated. Furthermore, increasing $T$ generally shows a substantial decrease in the development time for a given $U_{W}=E$, which is to be expect ed given the more rapid roll formation time that occurs at higher Rayleigh numbers.

\subsection{Charge and velocity uctuations}

In section 6, it was shown that in the stretched roll regime (regime B), as the top wall velocity increases, the roll development time increases, and for high values of $U_{W}$, charge and velocity magnitude uctuations can decrease as is also apparent through observation of Figs. $7(a)$ and $7(b)$. Temporal variations in space charge and velocity can be of critical importance in charge injection systems where there is a desire to predict time dependent charge and velocity distributions [22]. Therefore, we further analyse variations in space charge with time, in the context of this fundamental forced electro-convective problem. This is done here by monit oring the change in space charge with time at a xed control volume. Whilst averaging spatially across the domain as was done in section 6 gives a better statistical understanding of temporal changes in the space charge, it does not allow for an investigation of variations in charge from one roll to the next. This can 
be of vital importance in understanding sudden changes in space charge which occur between two electrodes, which in the context of charge injection atomizers, could lead to unforeseen drops in spray speci c charge.

The control volume is located in the centre of the domain lengthwise, and the result is shown in gure 9 for thre di erent vertical locations, for the conditions speci ed in the caption. Concentrating on the case of $y=0.5 \mathrm{~d}$, the inst ant in time where the control volume charge is low indicates a point in time in the central region of the roll where little charge is present. The top wall adds a horizont al velocity component which shifts a given roll over the control volume in time, hence resulting in a local increase in charge, as the highly charged layer between rolls translates along the domain. At a vertical location closer to the emitting electrode, it is clear that the 'pulse-width' of charge is much higher and this is because the cont rol volume is located close to the emitter where the degree of charge strati caton is less severe, as is also clear through the contour plots presented in section 4:2. At the top of the domain, close to the collecting electrode, the charge pulse-width increases again and this is due to the impingement of charge on the top surface which creates longitudinal spreading.

With respect to EHD Couette ow, it is of interest to ascertain whether the temporal variation in the space charge at a point is mostly driven by the translational velocity of these rolls, which generally scales with $U_{W}=E$ or $R e=R e_{E}$, or if there is an additional dependence, such as a dependence due to the shifting and displacement of rolls, which may be able to overcome the imposed convection.

Figure 10(a) shows that over a wide range of $T$, the temporal variation in the form of a frequency of charge uctuation, is heavily driven by the ratio of $U_{W}=E$ or $R e=R e_{E}$, given the near linear relationship that occurs over a wide range $T$ and $R e=R e_{E}$. Whilst more random uctuations in charge tend to occur at higher $\mathrm{T}$ values, the dominant 
frequency measured is in this instance related to the value of $U_{W}$.

Plotting the frequency of charge uctuations vs the body force term $\mathrm{Gr}_{\mathrm{E}}=\mathrm{Re}^{2}$ as shown in Fig. 10(b), reveals the relationship between the charge uctuation frequency and the forced convection body force term $\mathrm{Gr} \mathrm{e}_{\mathrm{E}}=\mathrm{Re}^{2}$. For these conditions, and for a given $T$ value, an increase in the charge uctuation frequency occurs with a decrease in the body force term. As shown in Fig. 10(a) this is largely at tributed to the increase in $\mathrm{Re}$ which is required for higher $\mathrm{T}$ numbers in order for a roll to occur. However, from the gradient of each of the $T$ curves, one can deduce that there is a secondary e ect of the $T$ number on the charge uctuation frequency for a $x e d \mathrm{Gr}_{E}=\operatorname{Re}^{2}$. This seems to be particularly true for lower values of $\mathrm{Gr}_{\mathrm{E}}=\mathrm{Re}^{2}$ where an increase in $\mathrm{T}$ can result in a substantially higher value of the charge uctuation frequency.

These results further show the impact of forced convection parameters on the dynamics of EHD roll structures, showing in particular how a forced velocity can take precedence over electrodynamic inputs into the ow.

\section{Conclusions}

This paper has provided new insights into the roll structure characteristics of a fundamental two-dimensional electro-convective Couette ow. Thre regimes have been identi ed, being a traditional single or double roll structure as in free convection, a stretched roll structure, and a regime where no roll structures are present and a solution is analytically possible. An increase in the upper wall velocity $U_{W}$ re-distributes the mean charge and velocity distribution betwen the two electrodes and for very high levels of $U_{W}$, uctuations in charge and velocity in the domain are dampened. The mean development time of the ow is dictated both by the T number and the upper wall velocity such that a stretched roll regime is characterized with an increase in the 
development time with an increase in $\bigcup_{W}$. Further analysis of temporal space charge variations at localized points in the domain show that the upper wall velocity is a dominating factor in dictating time dependent space charge however an increase in the electrical Rayleigh number can also have a secondary impact. Future work in forced electro-convection should include extension of this analysis to thre-dimensional ows, analysis of forced electro-thermal convection, relevant in heat transfer enhancement of industrial heat exchangers while also simulation of complex geometries. 


\section{Refer ences}

[1] P. A. Vazquez, G. E. Gheorgiou, and A. Castellanos. Numerical analysis of the stability of the electrohydrodynamic (ehd) electroconvection between two plates. J.Phys. D, 41, 2008.

[2] A Castellanos. Basic Concepts and Equations in Electrohydrodynamics in : Electrohydrodynamics 1st ed. (editor: Castellanos, A). Springer-Verlag, 1998.

[3] P.H. Traore, A.T. Perez, D. Koulova, and H. Romat. Numerical modelling of nite-amplitude electro-thermo-convection in a dielectric liquid layer subjected to both unipolar injection and temperature gradient. Journal of Fluid Mechanics, 658:279\{293, 92010.

[4] N. Kasayapanand and T. Kiatsiriroat. Numerical modeling of the electrohydrodynamic e ect to natural convection in vertical channels. International Communications in Heat and Mass Transfer, 34:162\{175, 2007.

[5] A.G. Bailey. The theory and practice of electrostatic spraying. Atomization Spray Technol., $2: 95\{134,1986$.

[6] AJ Kelly. The electrostatic atomization of hydrocarbons. Journal of the Institute of Energy, $57: 312\{320,1984$

[7] Kim K and Turnbull J. Generation of charged drops of insulating liquids by electrostatic spraying. Journal of Applied Physics, 47:1964\{1969, 1976.

[8] J. S. Shrimpton and A. J. Yule. Electrohydrodynamics of charge injection atomization: Regimes and fundamental limits. Atomization and Sprays, 12:173\{190, 2003.

[9] J. H. Davidson and P. J. McKinney. Turbulent mixing in a barbed plate-to-plate electrostatic precipitator. Atmos. Environ., 23:2093\{2107, 1989.

[10] J. H. Davidson and P. J. McKinney. Ehd ow visualization in the wire-plate and barbed plate electrostatic precipitator. IEEE Transactions on industry applications, 27:154\{160, 1991.

[11] Morita Y. Yamamoto T., Fujishuma H., and Okubo M. Threedimensional ehd simulation for point corona electrostatic precipitator based on laminar and turbulent models. Journal of Electrostatics, 64:628\{633, 2006.

[12] Joseph M. Crowley. Fundamentals of Applied Electrostatics. Laplacian Press, 1999.

[13] A. Castellanos. Coulomb-driven convection in electrohydrodynamics. IEEE Trans. Elect. Insulation, 26:1201\{1215, 1991.

[14] Atten P. Electrohydrodynamic instability and motion induced by injected space charge in insulating liquids. IEEE Transactions on Dielectrics and Electrical Insulation, 3:1\{17, 1996. 
[15] Schneider J. M. and Watson P. K. Electrohydrodynamic stability of space-charge-limited currents in dielectric liquids. i. theoretical study. Physics of Fluids, 13:1948\{ 1954, 1970.

[16] Hop nger E. J. and J. P. Gosse. Chargetransport by self-generated turbulence in insulating liquids submitted to unipolar injection. Physics of Fluids, 14:1671\{1682, 1971.

[17] J. Wu, P. Traore, and C. Louste. An e cient nite volume method for electric eldspace charge coupled problems. Journal of Electrostatics, 71(3):319 \{ 325, 2013. Journal of ELECTROSTATICS, Electrostatics 2013 12th International Conference on Electrostatics.

[18] Philippe Traore and Jian Wu. On the limitation of imposed velocity eld strategy for coulombdriven electroconvection ow simulations. Journal of Fluid Mechanics, 727, 72013.

[19] J. Wu, P. Traore, C. Louste, D. Koulova, and H. Romat. Direct numerical simulation of electrohydrodynamic plumes generated by a hyperbolic blade electrode. Journal of Electrostatics, 71(3):326 \{331, 2013. Journal of ELECTROSTATICS, Electrostatics 2013 12th International Conference on Electrostatics.

[20] P. A. Vazquez, G. E. Gheorgiou, and A. Castellanos. Characterization of injection instabilities in electrohydrodynamics by numerical modelling: comparison of particle in cell and ux corrected transport methods for electroconvection between two plates. J.Phys. D, 39, 2006.

[21] A. Kourmatzis and J.S. Shrimpton. Electrohydrodynamic inter-electrode ow and liquid jet characteristics in charge injection atomizers. Experiments in Fluids, 55(3), 2014.

[22] John Shrimpton. Charge Injection Systems: Physical Principles, Experimental and Theoretical Work. Springer-Verlag, 2009.

[23] R. M. Thaokar and V. Kumaran. Electrohydrodynamic instability of the interface between two uids con ned in a channel. Physics of Fluids, 17(8):\{, 2005.

[24] A.A. Mohamed, E.F. Elshehawey, and M.F. El-Sayed. Electrohydrodynamic stability of two superposed viscous uids. Journal of Colloid and Interface Science, 169(1):65 \{ 78, 1995.

[25] J.L. Lara, A. Castellanos, and F. Pontiga. Stability analysis of taylor-couette ow subjected to a weak unipolar injection of charge. IEEE Annual Report-Conference on Electrical Insulation and Dielectric Phenomena, pages 706\{ 709, 1997.

[26] Nabile T. M. Eldabe. Electrohydrodynamic stability of two superposed elasticoviscous liquids in plane couette ow. Journal of Mathematical Physics, 28(11):2791\{2800, 1987.

[27] Juan L. Lara, Francisco Pontiga, and Antonio Castellanos. Stability analysis of a taylorcouette ow of insulating uid subjected to radial unipolar injection of charge. Physics of Fluids, 
$10(12): 3088\{3098,1998$

[28] J.L. Lara, A. Castellanos, and F. Pontiga. In uence of unipolar charge injection on the stability of plane poiseuille ow. 12th International Conference on Conduction and Breakdown in Dielectric Liquids, pages 118\{121, 1996.

[29] Juan L. Lara, Antonio Castellanos, and Francisco Pontiga. Destabilization of plane poiseuille ow of insulating liquids by unipolar charge injection. Physics of Fluids, 9(2):399\{ 406, 1997.

[30] A. Castellanos and N. Agrait. Unipolar injection induced instabilities in plane parallel ows. IEEE Transactions on Industry Applications, 28:513\{519, 1992.

[31] Koulova Dantchi, Traore Philippe, Romat Hubert, Wu Jian, and Louste Christophe. Numerical simulations of electro-thermo-convection and heat transfer in $2 \mathrm{~d}$ cavity. Journal of Electrostatics, 71(3):341 \{ 344, 2013. Journal of ELECTROSTATICS, Electrostatics 2013 12th International Conference on Electrost atics.

[32] Jian Wu and Philippe Traore. A nite-volume method for electro-thermoconvective phenomena in a plane layer of dielectric liquid. Numerical Heat Transfer, Part A: Applications, 68(5):471\{500, 2015.

[33] J. Wu, P. Traore, C. Louste, A. T. Perez, and P.A. Vazquez. Heat transfer enhancement by an electrohydrodynamic plume induced by ion injection from a hyperbolic blade. IEEE International Conference on Liquid Dielectrics, pages 1\{4, 2014.

[34] Shrimpton J. and Kourmatzis A. Direct numerical simulation of forced ow dielectric ehd within charge injection atomizers. IEEE Trans. Die. Elec. Ins., 17:1838\{ 1845, 2010.

[35] A. Kourmatzis and J. S. Shrimpton. Turbulent three-dimensional dielectric electrohydrodynamic convection between two plates. Journal of Fluid Mechanics, 696:228\{262, 42012.

[36] H K Versteeg and W Malalasekera. An introduction to computational uid dynamics. Longman scienti c and technical, 1995.

[37] M. Davoudabadi, J.S. Shrimpton, and F. Mashayek. On accuracy and performance of highorder nite volume methods in local mean energy model of non-thermal plasmas. Journal of Computational Physics, 228(7):2468 \{ 2479, 2009.

[38] R. Chicon, A. Castellanos, and E. Martin. Numerical modelling of coulomb-driven convection in insulating liquids. J. Fluid Mech., 344:46\{66, 1997.

[39] J.C. Lacroix, Atten P., and Hop nger E.J. Electro-convection in a dielectric liquid layer subjected to unipolar injection. Journal of Fluid Mechanics, 69:539\{564, 1975. 
[40] A.R.H. Rigit and JohnS. Shrimpton. Estimation of the diamet ercharge distribution in polydisperse electrically charged sprays of electrically insulating liquids. Experiments in Fluids, 46(6):1159\{ 1171,2009 


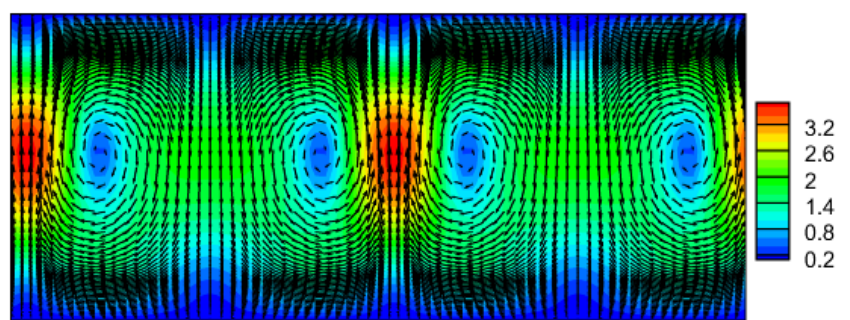

(a)

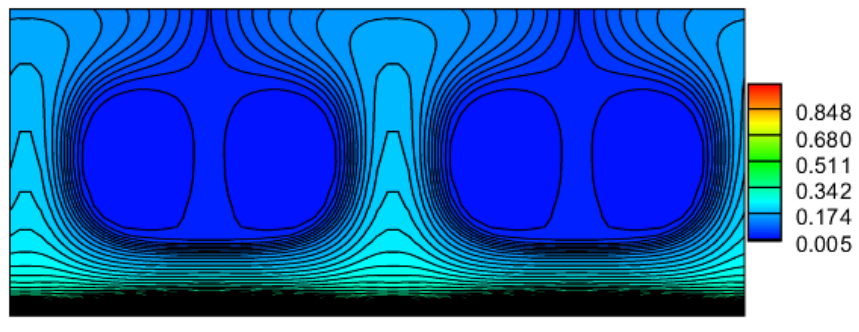

(b)

Figure 1. Flow distribution (a) colored with non-dimensional velocity magnitude contours where $L=2.4 d$, the maximum velocity magnitude is normalized with the free convection drift velocity $\mathrm{E}_{0}$ and $\mathrm{E}_{0}=\mathrm{V}_{0}=\mathrm{d}_{0}$. In (b) the non-dimensional charge distribution $Q=Q_{B}$ is shown. Both plots are for $T=200, C=10, M=10$ as in [1]. 


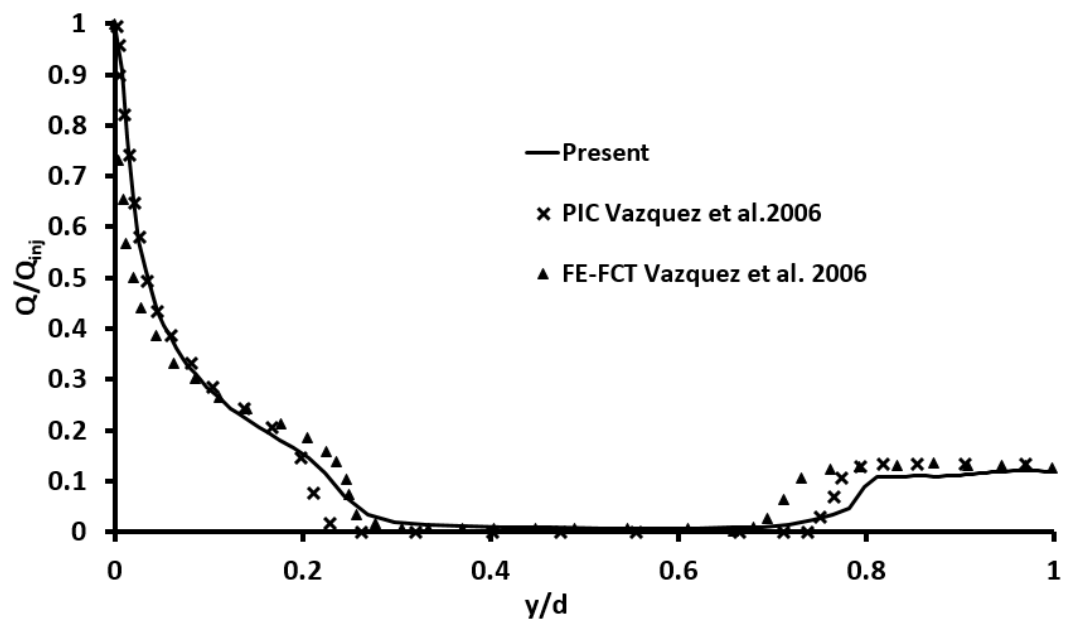

Figure 2. $Q$ vs vertical position for $T=200, C=10, M=10$ case through centre of roll 


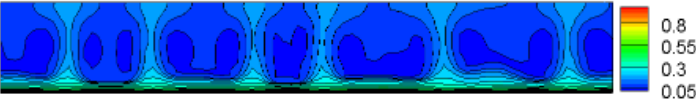

(a) $T=500, R e=R e_{E}=0: 5$

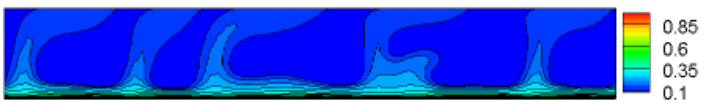

(c) $\mathrm{T}=500, \mathrm{Re}=\mathrm{Re}_{\mathrm{E}}=5$

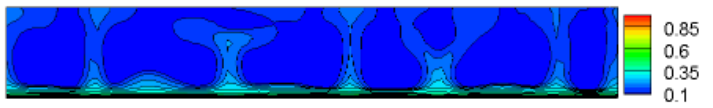

(e) $\mathrm{T}=750, \mathrm{Re}=\mathrm{ReE}=0: 7$

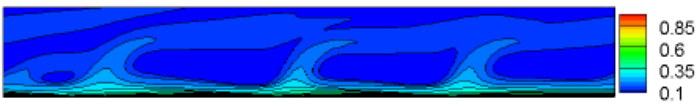

(g) $T=750, R e=R e_{E}=20$

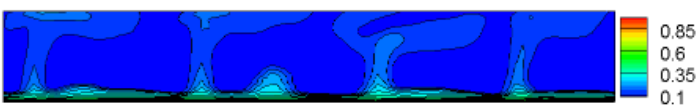

(i) $T=1500, R e=R e_{E}=5$

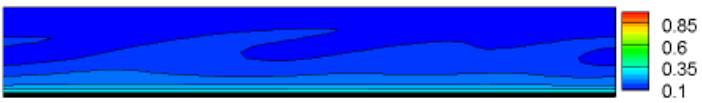

(k) $T=1500, R e=R e_{E}=40$

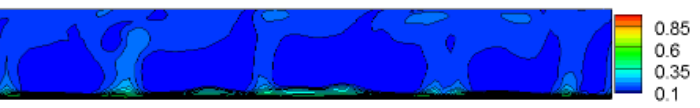

(m) $\mathrm{T}=3000, \mathrm{Re}=\mathrm{ReE}_{\mathrm{E}}=5$

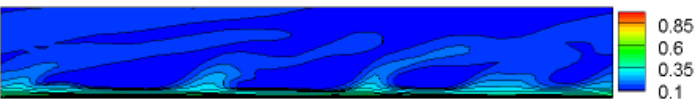

(o) $\mathrm{T}=3000, \mathrm{Re}=\mathrm{R} e_{E}=50$

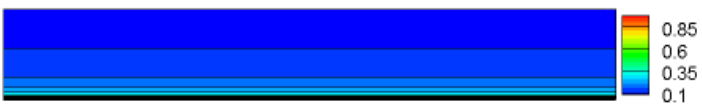

(q) $T=1500, R e=R e_{E}=45$

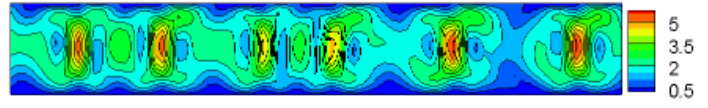

(b) $T=500, R e=R e_{E}=0: 5$

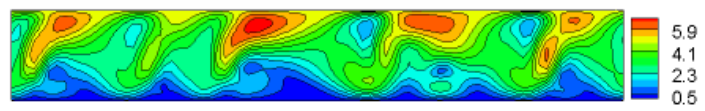

(d) $T=500, R e=R e_{E}=5$

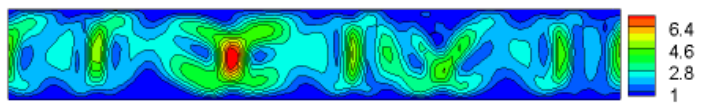

(f) $T=750, R e=R e_{E}=0: 7$

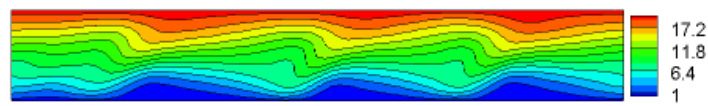

(h) $\mathrm{T}=750, \mathrm{Re}=\mathrm{Re} \mathrm{e}_{\mathrm{E}}=20$

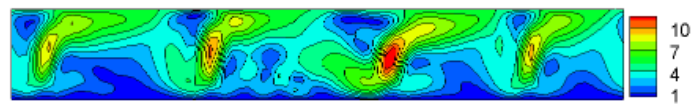

(j) $T=1500, R e=R e_{E}=5$

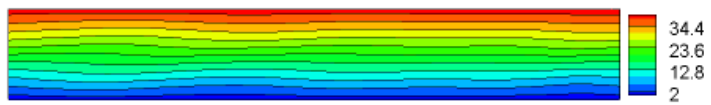

(I) $T=1500, R e=R e_{E}=40$

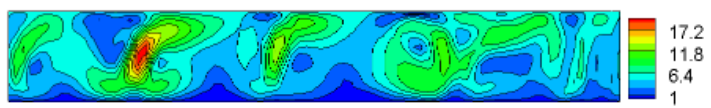

(n) $T=3000, R e=R e_{E}=5$

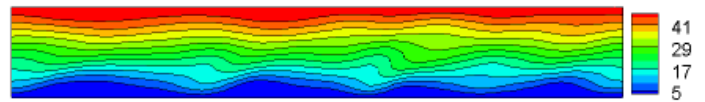

(p) $\mathrm{T}=3000, \mathrm{Re}=\mathrm{ReE}=50$

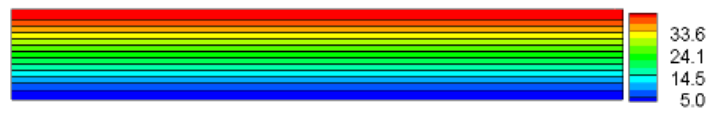

(r) $T=1500, R e=R e_{E}=45$

Figure 3. Normalized space charge distribution $Q=Q_{B}$ (left) and normalized velocity magnitude $U_{m a g}=E$ (right) as a function of $T$ and upper wall velocity 


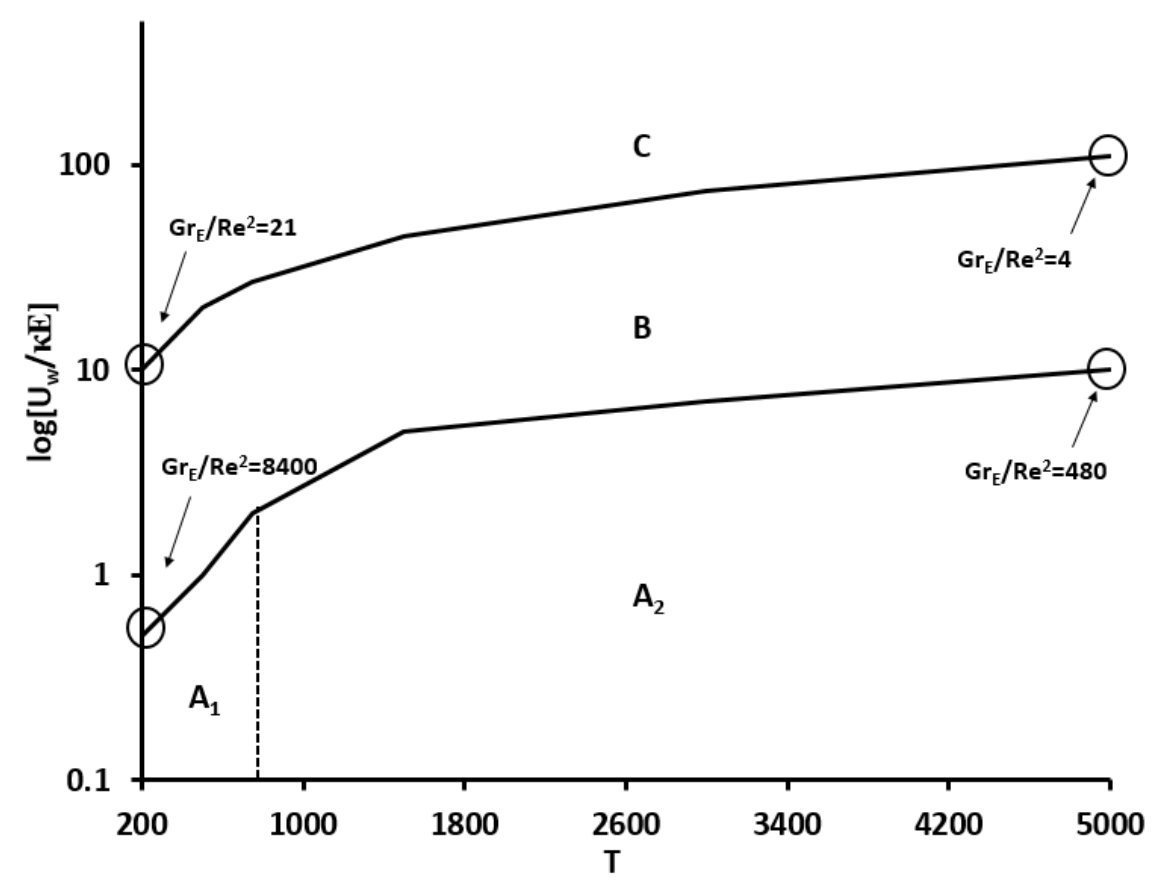

Figure 4. Electroconvective roll structure map on a log-linear scale as a function of non-dimensional wall velocity and $\mathrm{T}$ parameter for $\mathrm{C}=10$ and $\mathrm{Re}_{\mathrm{E}}=1$ 


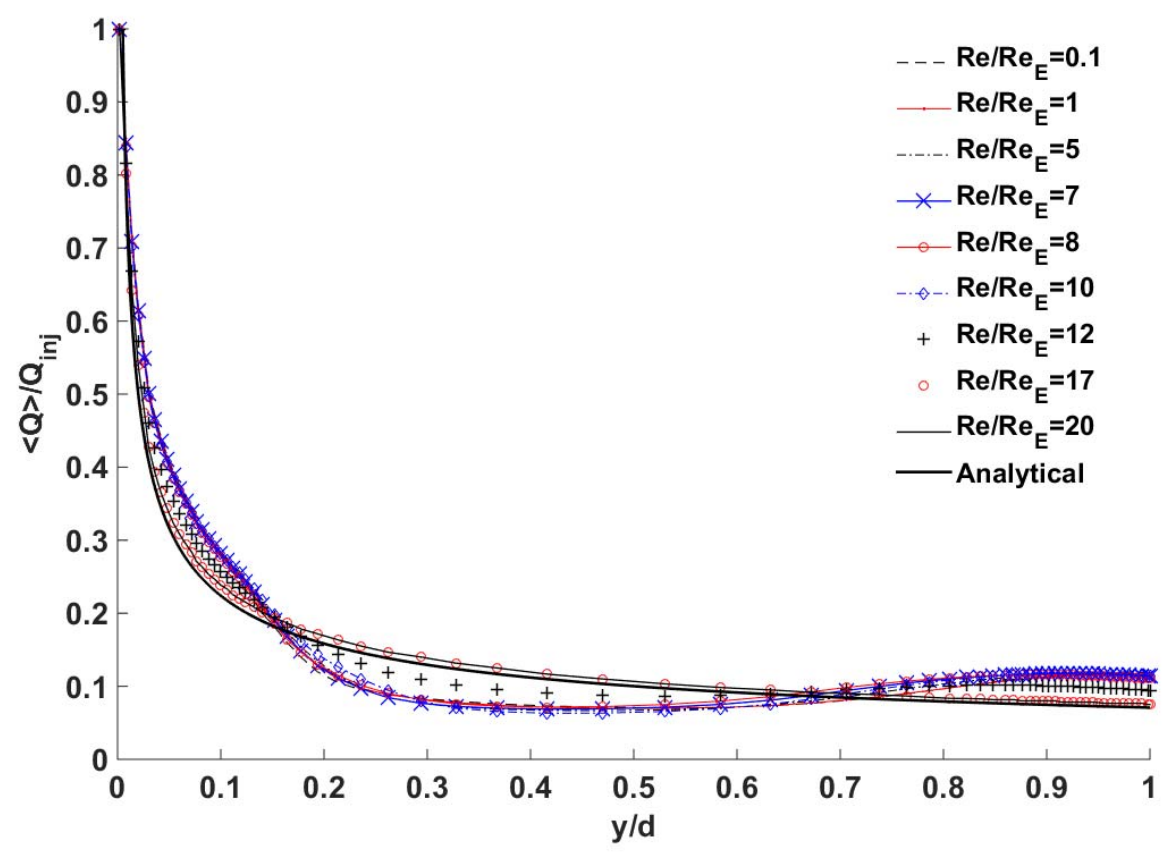

(a) Charge distribution

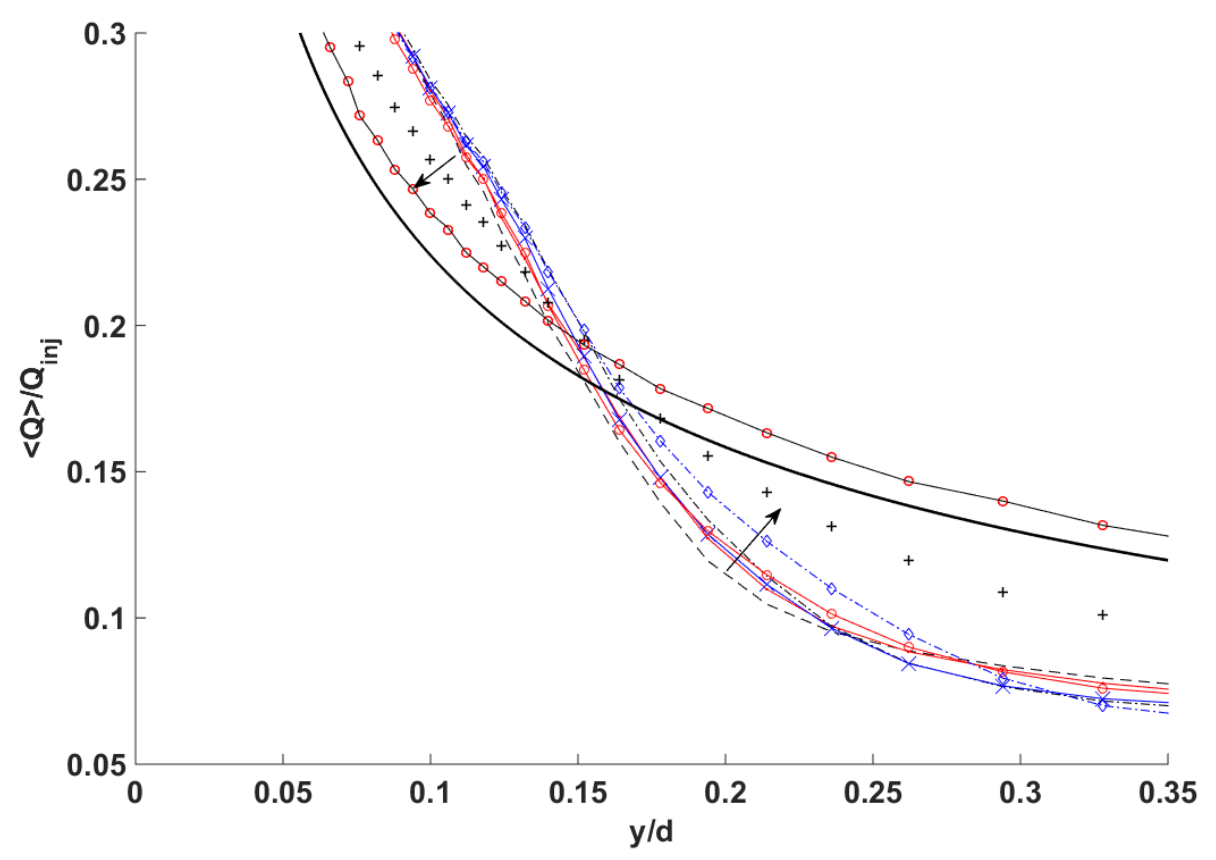

(b) Magni ed Charge distribution

Figure 5. Non-dimensional space charge vs. non-dimensional vertical distance as a function of wall velocity for $T=500, C=10$ and $R_{E}=1$ 


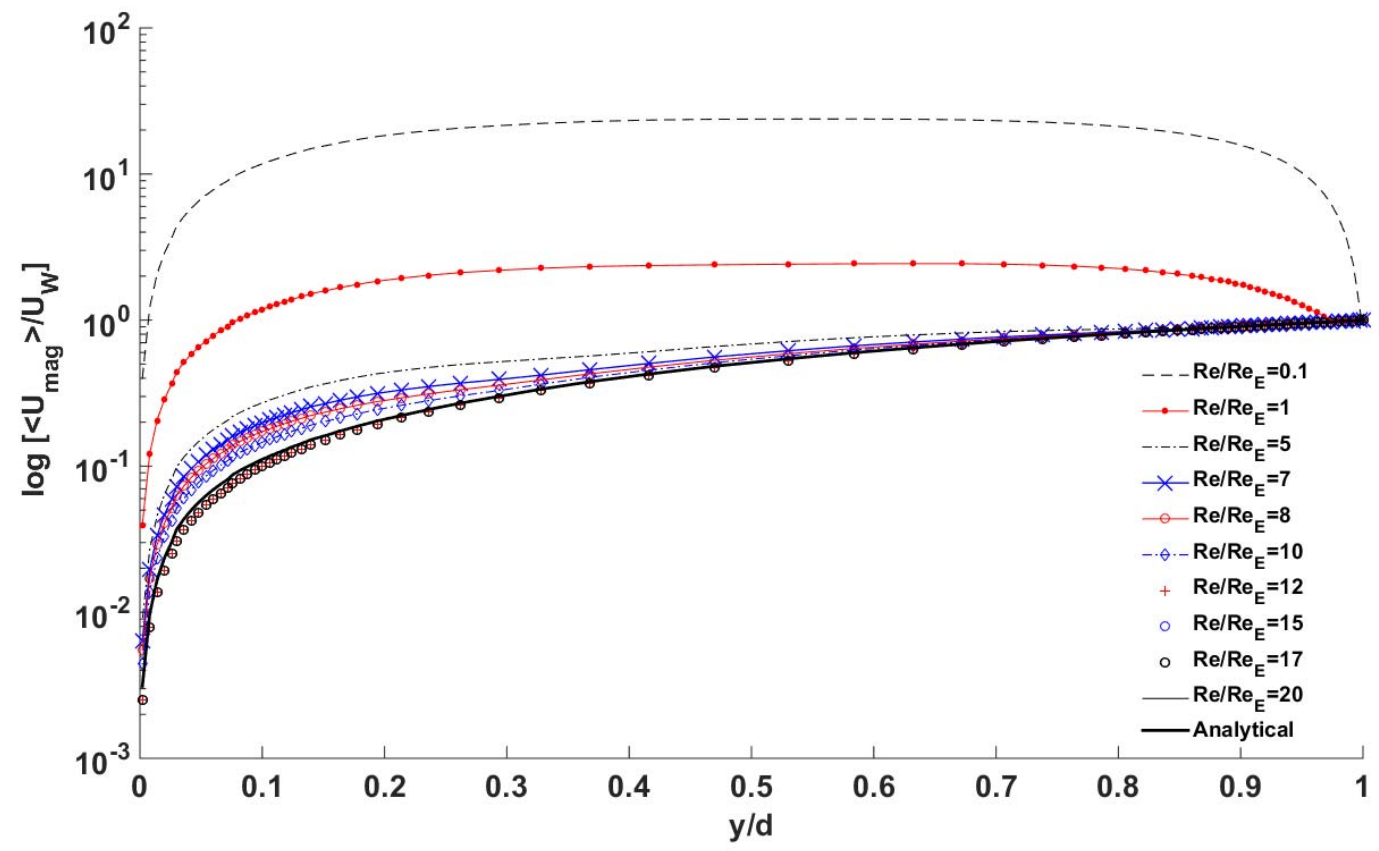

Figure 6. Non-dimensional velocity magnitude vs. non-dimensional vertical distance as a function of wall velocity for $T=500, C=10$ and $R e_{E}=1$. Note: vertical axis is on a logarithmic scale, dimensionless analytical solution for Couette ow is a straight line from the origin to $(1,1)$. 


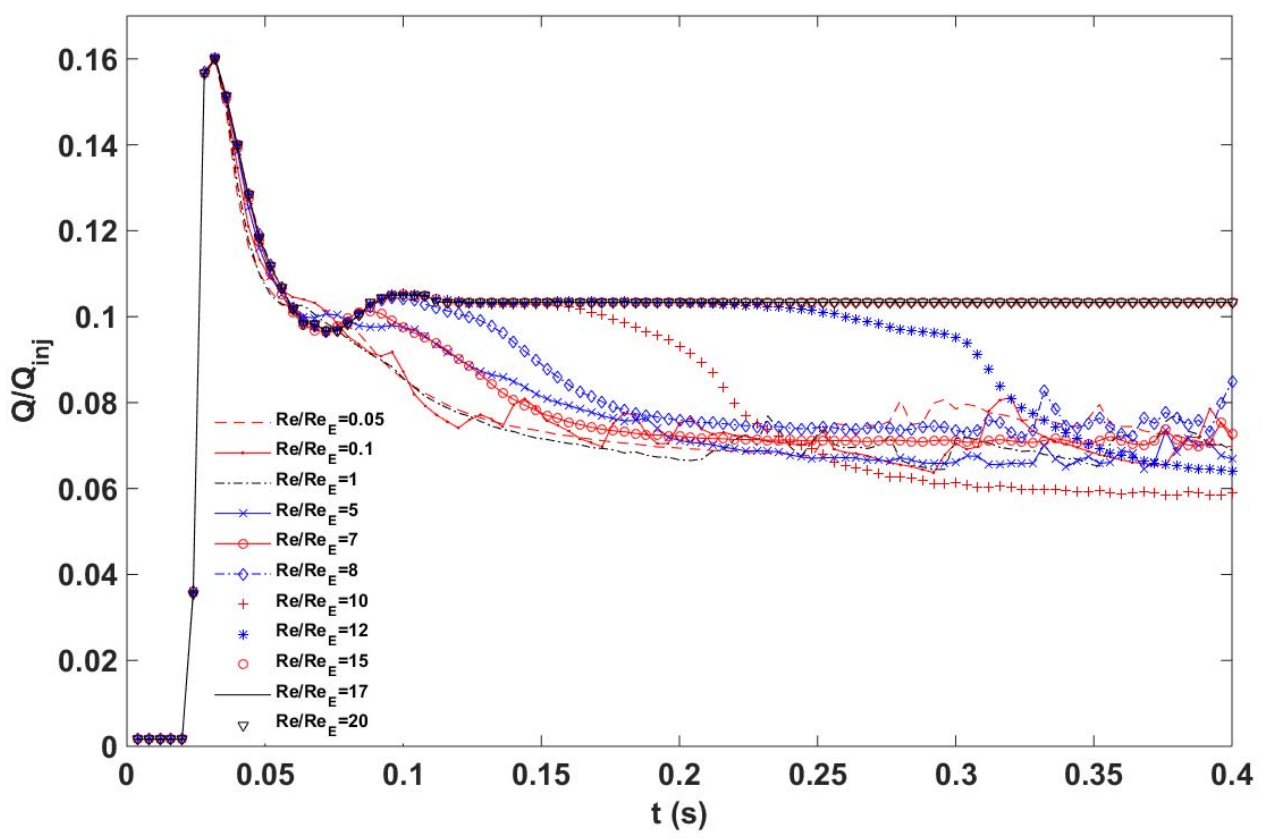

(a) Space Charge

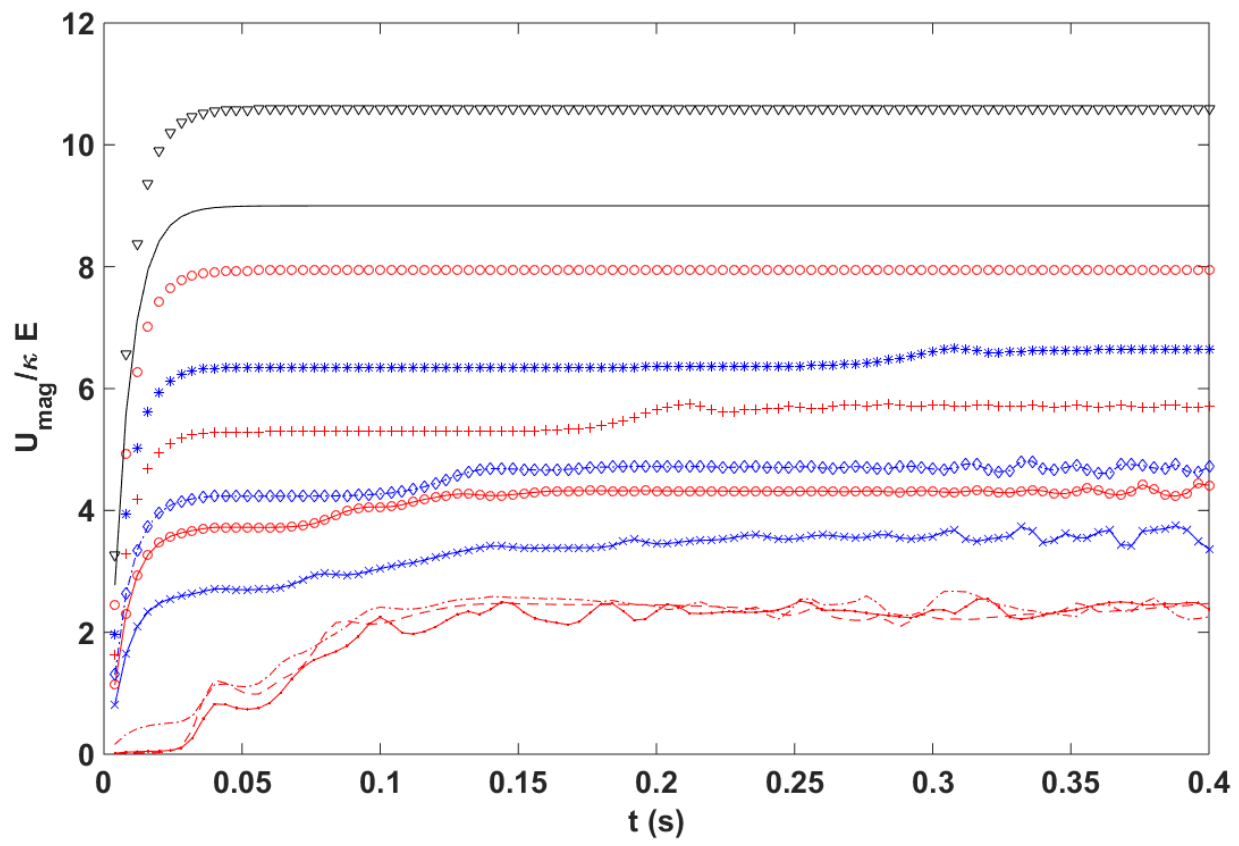

(b) Velocity Magnitude

Figure 7. Mean non-dimensional space charge and velocity magnitude at $y=0.5 \mathrm{~d}$ vs. time as a function of wall velocity for $T=500, C=10$ and $R_{E}=1$ where ${ }_{d}=0: 06 \mathrm{~s}$ 


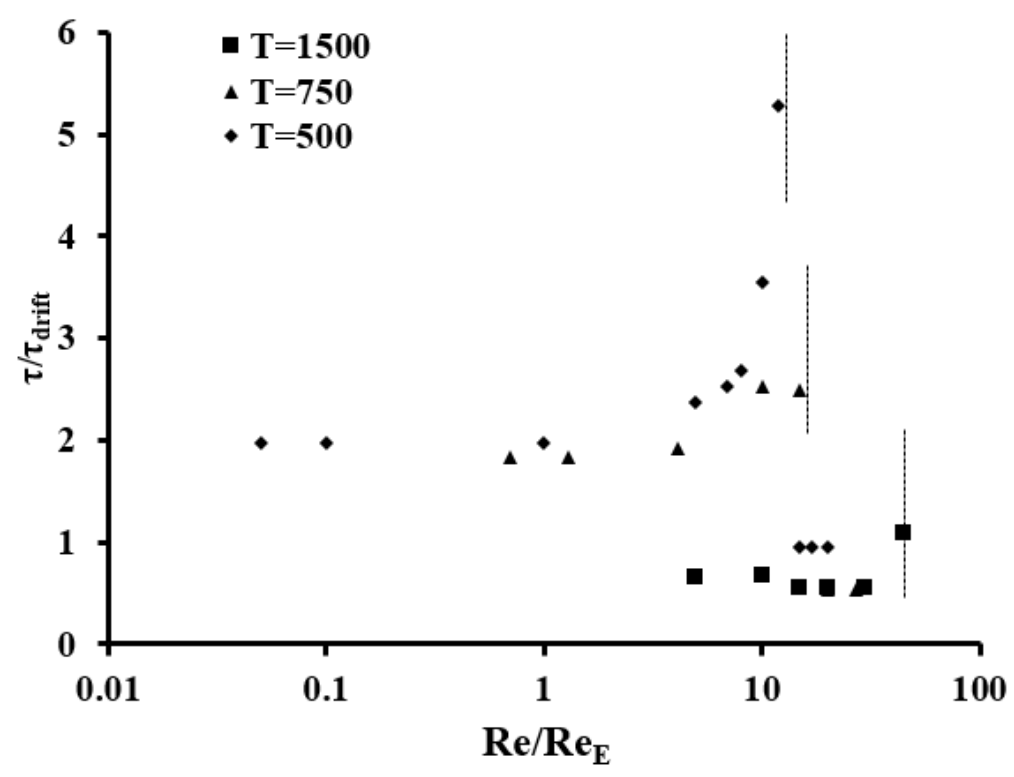

Figure 8. Steady state development time normalized by ionic drift time-scale for a variety of $T$ values plotted vs. $R e=R e_{E}$ where vertical lines indicate nal transition to a state of no-rolls 


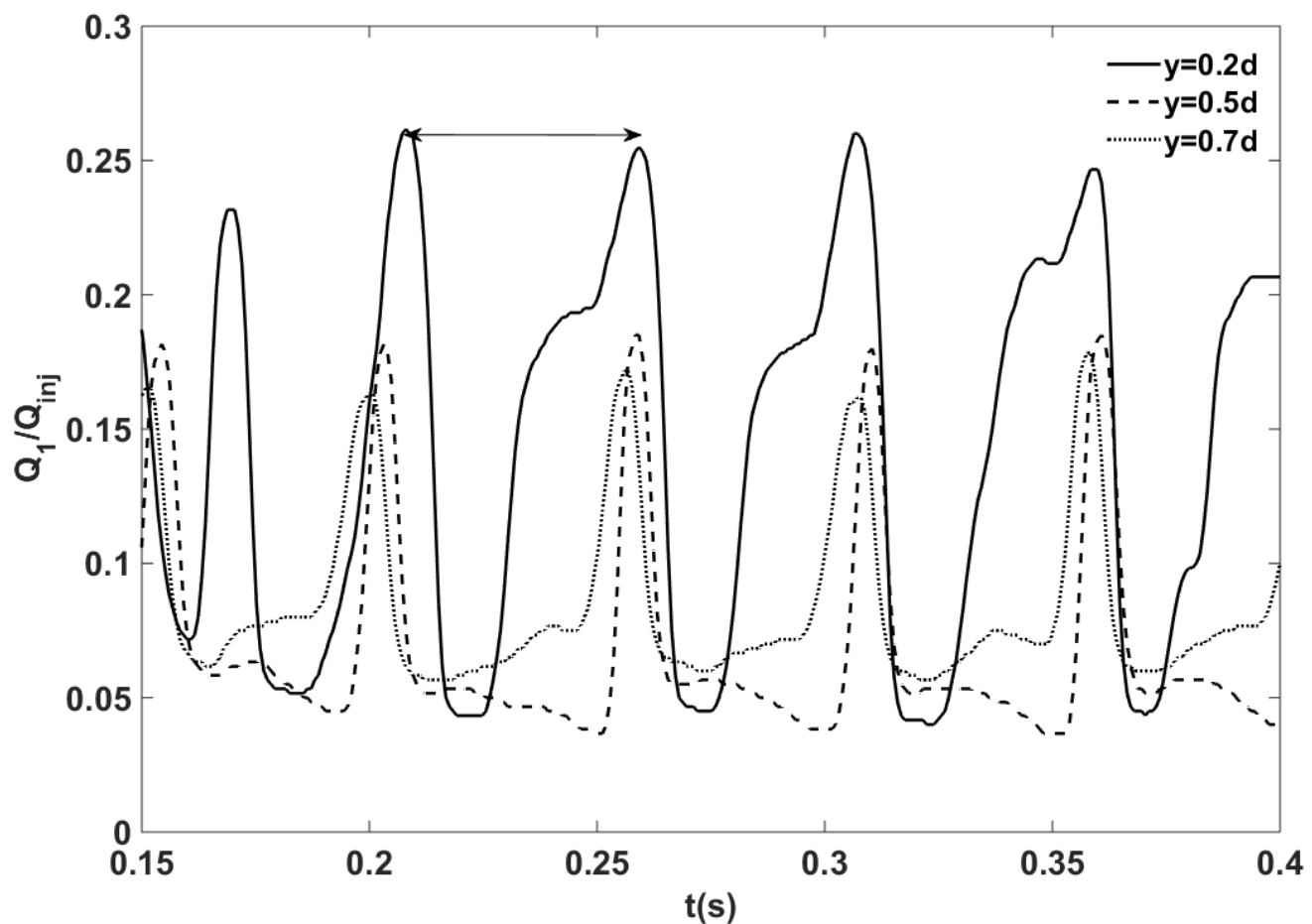

Figure 9. Non-dimensional space charge at centre of the domain vs. time at di erent vertical locations for $T=500$ and $U_{W}=E=5$ where ${ }_{d}=0: 06 s$ 


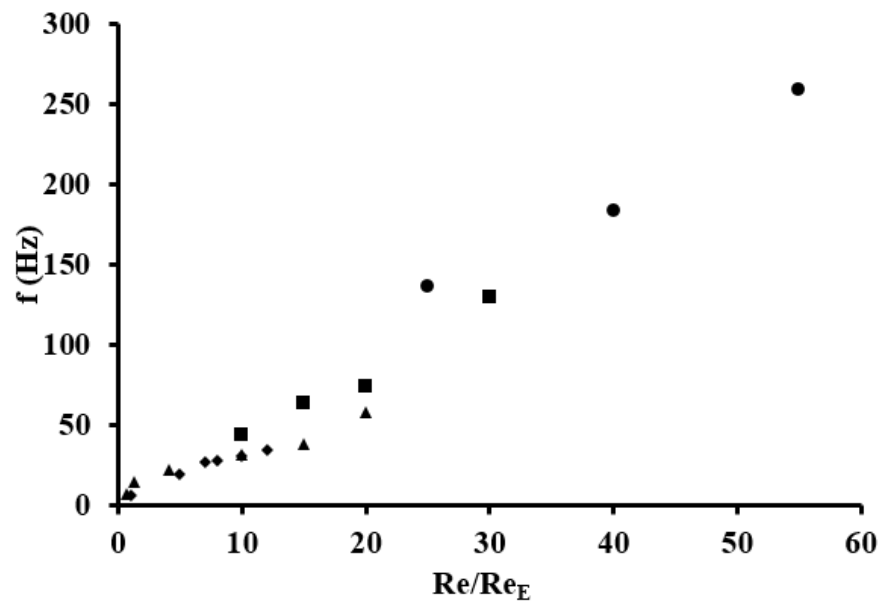

(a)

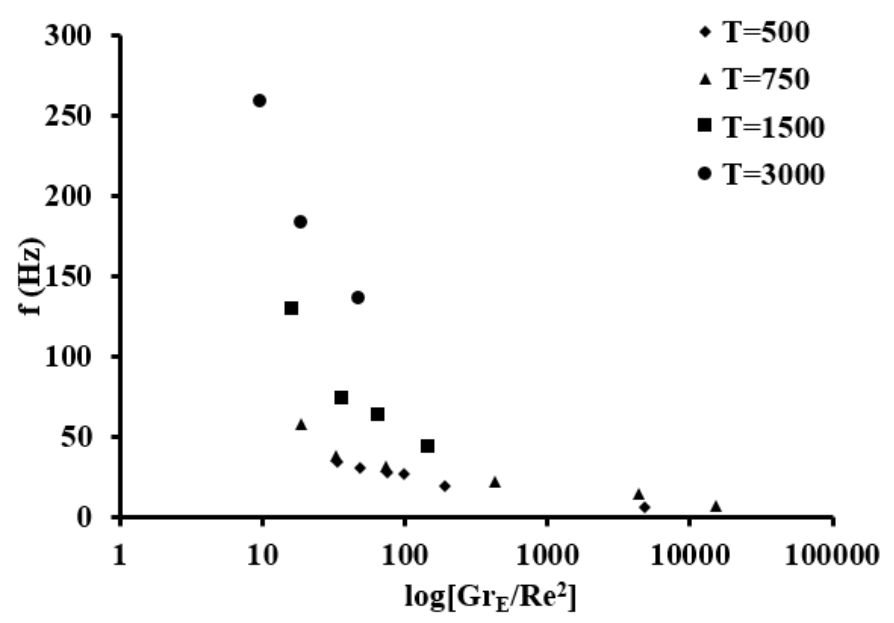

(b)

Figure 10. Frequency of charge variation in xed control volume at the centre of the domain vs $\operatorname{Re}=\operatorname{Re}_{E}$ (a) and $\mathrm{Gr}_{E}=\operatorname{Re}^{2}$ (b) and for a variety of $\mathrm{T}$ values 